\title{
Reverse biased nanocrystalline graphite (NCG)/p-Si schottky junction for methane gas sensor
}

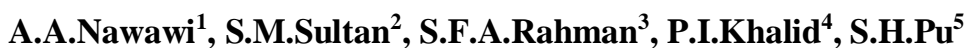 \\ ${ }^{1,2,3,4}$ School of Electrical Engineering, Faculty of Engineering, Universiti Teknologi Malaysia (UTM), Malaysia \\ ${ }^{5}$ University of Southampton Malaysia Campus, Malaysia
}

\section{Article Info \\ Article history: \\ Received Dec 25, 2018 \\ Revised Mar 28, 2019 \\ Accepted Apr 22, 2019}

\section{Keywords:}

Barrier height

Methane

Nanocrystalline graphite

Reverse biased

Schottky diode

\begin{abstract}
An investigation on the effect of the reverse biased operation of NCG/p-Si Schottky contact during methane gas exposure at room temperature has been presented. The experimental results show the larger current shift at the reverse bias operation, compared to the forward bias by exposing to methane gas. This can be attributed to the adsorption of methane gas into the metal surface layer and produces a negative charge at the junction, thus reduces the barrier height of the device. The reverse barrier height was calculated under the reverse bias condition, demonstrated the value decreased from 0.58 $0.53 \mathrm{eV}$ towards a higher concentration of methane gas. The Schottky junction also affected by the increase in a free carrier when exposure to the reducing gas such as methane. Raman spectra are reported to be detected at $\mathrm{G}, \mathrm{D}$ and $2 \mathrm{D}$ band with the grain size $1.88 \mathrm{~nm}$ to exhibit single crystallite graphite properties. The results correlate well with the 3D AFM scans reveal the RMS surface roughness of 1.1 to $2.8 \mathrm{~nm}$.
\end{abstract}

Copyright $(0) 2019$ Institute of Advanced Engineering and Science. All rights reserved.

\section{Corresponding Author:}

Suhana Mohamed Sultan,

School of Electrical Engineering,

Faculty of Engineering,

Universiti Teknologi Malaysia,

81310 UTM Johor Bahru, Malaysia.

Email: suhanasultan@utm.my

\section{INTRODUCTION}

With the development of nanofabrication technology and the advent of nanomaterials, many nanostructured devices been directed toward the development of gas sensor fabrication [1-4]. Historically, metal oxide semiconductor is preferred due to its response towards target gases, consequently increases the conductivity of the junction [5-8]. However, it requires high working temperature during the gas sensing operation. Recent years, the carbon nanomaterials have shown the unique properties in term of electrical, mechanical and optical in the area of gas sensing thus replacing the other well-established nanomaterials [4, 9-12].

Many authors have revealed the nanostructured Schottky diode demonstrated a lower reverse breakdown voltage in I-V characteristics [13-15]. This is due to the grains and sharp edges of the nanostructured surface, which produce strong local electric fields. The induced local electric fields at the sharp edge of nanostructures at the interface are a function of reverse bias voltage for a Schottky diode. Experimentally, Singh et al. [10] observed this phenomenon with Graphene/Silicon Schottky Diode. The device exhibited a high reverse bias dependent gas detection sensitivity, which enables molecular adsorption on its surface to alter the graphene/Si interface barrier height.

Herein, the Schottky diode based devices using $\mathrm{p}$-Si as a substrate with nanocrystalline graphite (NCG) are demonstrated for the application of methane gas sensing at room temperature. Methane gases are harmful to the environment especially towards human health, if overexposure takes place [11]. Reviews have 
shown that carbon-based nanomaterials are a convenient material at room temperature sensing applications $[4,12]$. NCG has been described in various structural forms with high surface to volume ratios which result in good capabilities for gas detection [13]. The response of the device towards different concentration of methane gas was investigated by monitoring the changes of I-V characteristics. The sensor device exhibits a dynamic response upon exposure with target gas which is verified by the current shift of the reverse bias in I-V characteristics.

\section{RESEARCH METHOD}

The NCG/p-Si are fabricated based on previous work [20, 21]. The 6-inch of a p-type silicon wafer with a resistivity of 19-21 $\Omega$.cm has been utilized for this work. The cleaning process of wafer started with using fuming nitric acid to eliminate organic impurities for 10 minutes. A layer of a $\mathrm{SiO}_{2}$ thickness of $200 \mathrm{~nm}$ was deposited using Plasma-enhanced Chemical Vapor Deposition at $350^{\circ} \mathrm{C}$, then patterned using photolithography and Reactive Ion Etching. Subsequently, NCG film with 9nm-thickness was deposited onto the patterned wafer by using a PECVD process. The fabrication process flow of NCG/p-Si is described in Figure 1. After the fabrication of the devices, the nanostructured material films were characterized by applying several techniques such as Field Enhanced Scanning Electron Microscopy (FESEM), Atomic Force Microscopy (AFM), and Raman spectroscopy. The materials characterizations results will be presented in detail.

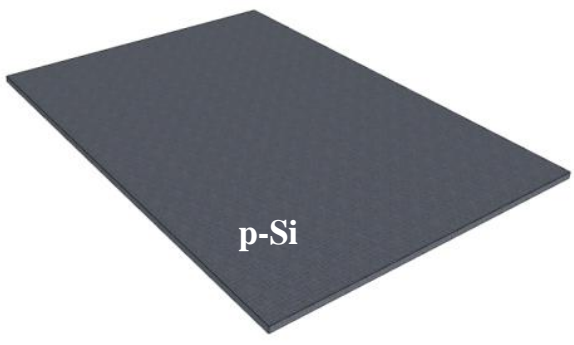

(a)

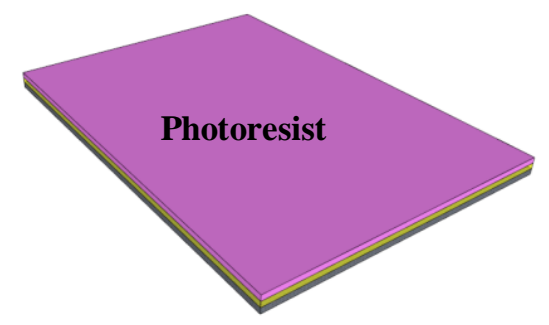

(c)

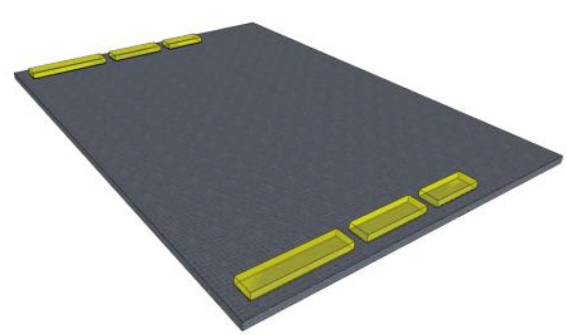

(e)

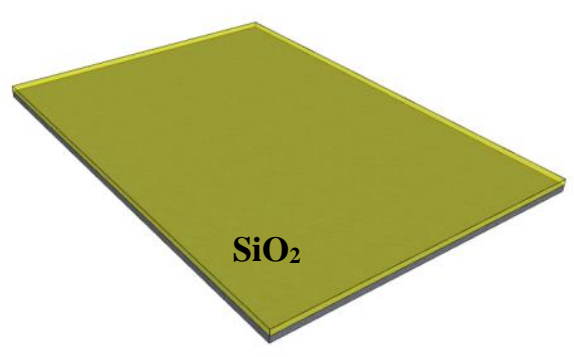

(b)

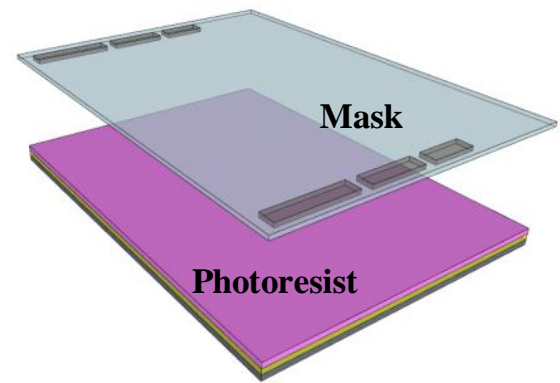

(d)

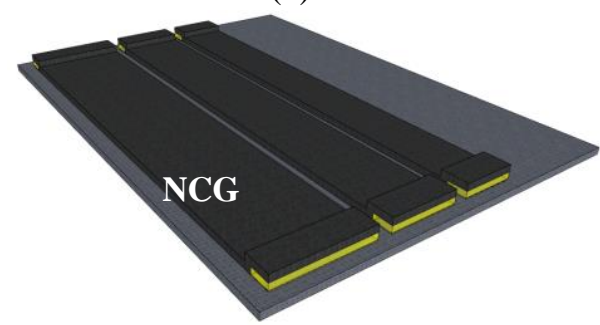

(f)

Figure 1. The processing steps of the NCG/p-Si fabrication, (a)Cleaning process of the substrate, (b)Deposition of 200nm $\mathrm{SiO}_{2}$ on p-Si using PECVD, (c)Spin coating the positive photoresist, (d) Mask alignment and exposure to the UV light, (e)Develop the pattern, etching the uncovered $\mathrm{SiO}_{2}$ using RIE,

(f)Deposition of NCG using PECVD process 


\section{RESULTS AND ANALYSIS}

\subsection{Material characterization}

Figure 2 shows the AFM scan of the three dimensional (3D) surface morphology NCG thin films deposited on $\mathrm{SiO}_{2} / \mathrm{p}$-Si substrates. The surface of NCG thin film is composed of having root-mean-square (RMS) surface roughness of approximately 1.1 to $2.8 \mathrm{~nm}$, which is believed to have a significant effect on gas interaction. The NCG film was examined using FESEM at 70k magnifications in Figure 3 to obtain the surface images which reveal a good agreement with other comparable films to indicate the nano-graphitic crystallites [15, 16]. Raman spectrum reveals the existence of the $G$ band $\left(1596 \mathrm{~cm}^{-1}\right)$ and $D$ band $\left(1353 \mathrm{~cm}^{-1}\right)$ in Figure 4, assigned to the sharp graphitic and amorphous carbon, respectively. The ratio of $I_{G}$ (4.13 a.u) and $I_{D}(9.66 \mathrm{a}$.u $)$ peaks, gives an indication of the grain size, $L_{a}$ using (1) which yields a value of $1.88 \mathrm{~nm}$ for this film [17].

$$
L_{a}=4.4 n m\left(\frac{I_{G}}{I_{D}}\right)
$$

where $I_{G}$ and $I_{D}$ is the intensity at the G and D band, respectively.

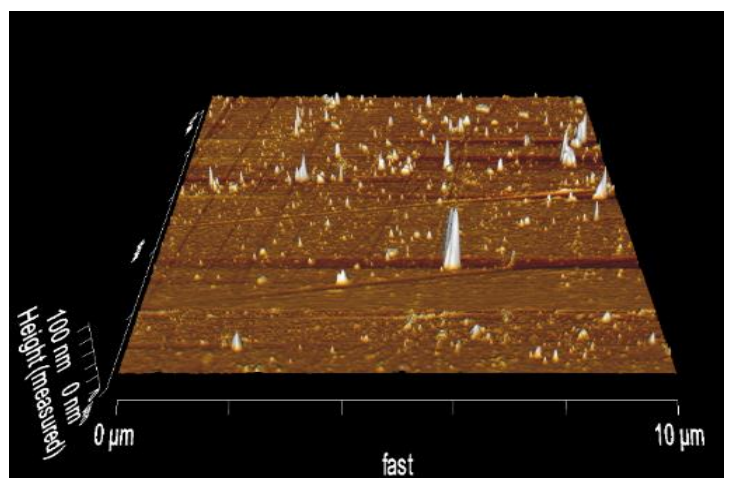

Figure 2. AFM 3D images of as-deposited NCG thin films on $\mathrm{SiO}_{2} / \mathrm{p}-\mathrm{Si}$

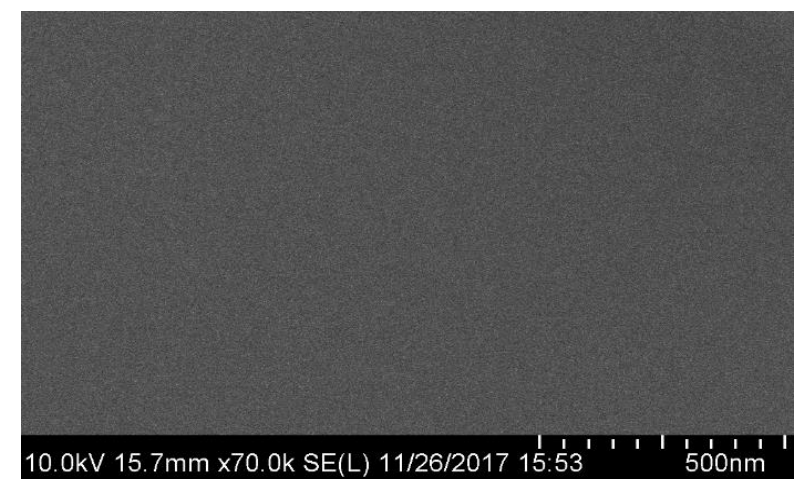

Figure 3. FESEM image of a NCG thin film 70K magnification with field emission gun at $10 \mathrm{kV}$ and a working distance of $15.7 \mathrm{~mm}$

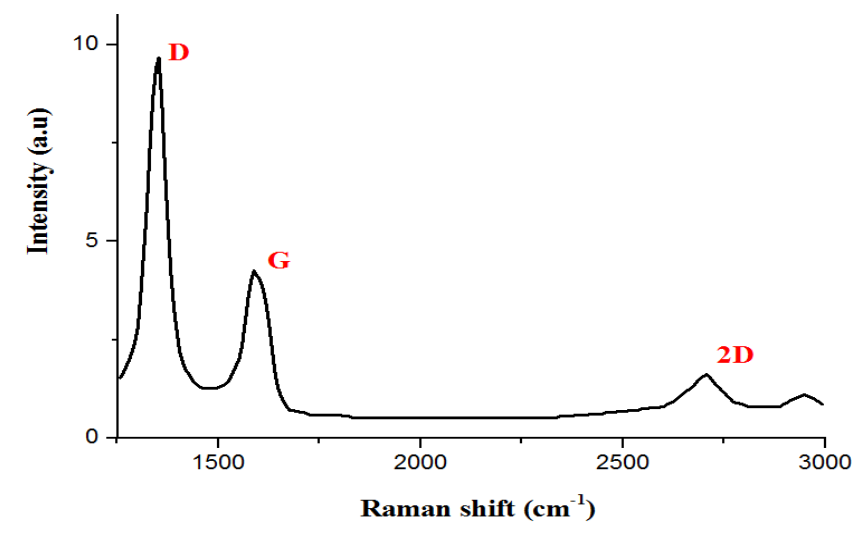

Figure 4. A Raman spectrum of the deposited NCG on a p-Si substrate

\subsection{Electrical and Gas Sensing Characterization}

Figure 5 shows the $\mathrm{I}-\mathrm{V}$ characteristics of the device in ambient air with various concentrations of methane, $\mathrm{CH}_{4}(25 \%, 50 \%, 75 \%$ and $100 \%)$ at room temperature. In the reverse bias, the fabricated sensor exhibits a lateral current shift, meanwhile, the forward bias operation remains similar to all exposure of $\mathrm{CH}_{4}$ gas. This result is almost identical to the finding reported by Shafiei et al. [13], which has the same reverse bias operation for Schottky diode gas sensing. Using metal-semiconductor theory, the Schottky I-V characteristics in the forward and reverse operations are given by (2) and (3) [24]. 


$$
\begin{aligned}
& I_{F}=S A^{*} T^{2} \exp \left(\frac{-q \emptyset_{b}}{k T}\right) \exp \left(\frac{q V_{F}}{\eta k T}\right) \text { for } V_{F}>3 k T / q \\
& I_{R}=S A^{*} T^{2} \exp \left(-\frac{q\left(\emptyset_{b(R E V)}-\sqrt{q \xi_{m} / 4 \pi \varepsilon_{S}}\right)}{k T}\right) \text { for } V_{R}>3 k T / q
\end{aligned}
$$

where $I_{F}$ is the forward current, $I_{R}$ is the reverse current, $S$ is the Schottky contact area, $A^{*}$ is the effective Richardson constant, $\mathrm{T}$ is the absolute temperature, $q$ is the charge constant, $\eta$ is the ideality factor, $V_{F}$ is the forward bias voltage, $\emptyset_{b}$ is the barrier height, $\varepsilon_{S}$ is the electric permittivity of $\mathrm{SiO}_{2}, \mathrm{k}$ is the Boltzmann constant and $\xi_{m}$ is the electric field at the interface of metal/semiconductor. $\xi_{m}$ is a function of the reverse bias voltage $V_{R}$ which is expressed by (4) [8],

$$
\xi_{m}=\sqrt{\frac{2 q N_{A}}{\varepsilon_{S}}\left(\left|V_{R}\right|+\psi_{b i}-\frac{k T}{q}\right)}
$$

where $\psi_{b i}$ is the built-in voltage and $N_{A}$ is the number of dopant in the silicon substrate. The changes in reverse barrier height, $\emptyset_{b(R E V)}$ of the sensor can be calculated by combining (3) and (4). Assuming the dominations of $V_{R}>5 \mathrm{~V}$ over the Schottky barrier, thus $V_{R} \gg \psi_{b i}-(\mathrm{kT} / \mathrm{q})$ [19].

$$
\ln \left(\frac{I_{R}}{S A^{*} T^{2}}\right)=-\frac{q \emptyset_{b(R E V)}}{k T}+\frac{q \alpha}{k T}\left|V_{R}\right|^{1 / 4}
$$

where,

$$
\alpha=\left(\frac{q^{3} N_{D}}{8 \pi^{2} \varepsilon_{S}}\right)^{1 / 4}
$$

By plotting $\ln \left(I_{R} / S A^{*} T^{2}\right)$ versus $\left|V_{R}\right|^{1 / 4}$ in Figure 6 , the value of $\emptyset_{b(R E V)}$ can be found by extracting the y-intercept of this plot. The value of $\emptyset_{b(R E V)}$ ranges from $0.58-0.53 \mathrm{eV}$ upon exposure to a various concentration of $\mathrm{CH}_{4}$ as represented in Table 1 .

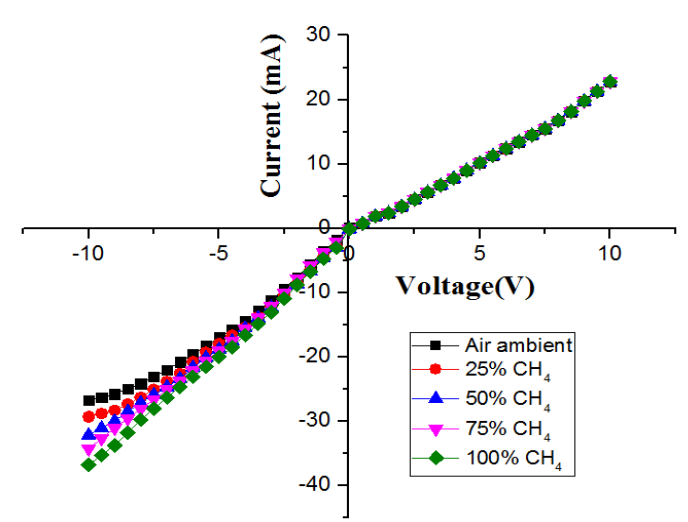

Figure 5. Plots of I-V characteristic of NCG/p-Si Schottky junction based sensor measured at air ambient and towards 25\%, 50\%, 75\% and 100\% methane concentration at room temperature

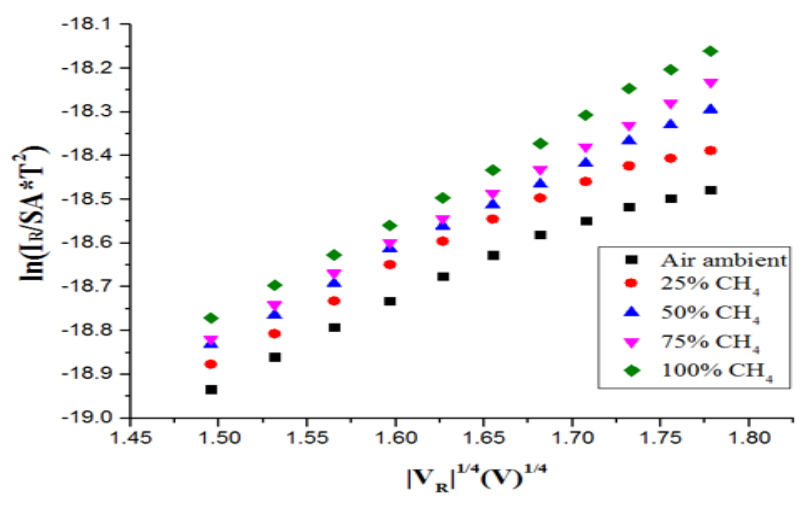

Figure 6. Plots of $\ln \left(\mathrm{I}_{\mathrm{R}} / \mathrm{SA} \mathrm{T}^{2}\right)$ versus $\left|\mathrm{V}_{\mathrm{R}}\right|^{1 / 4}$ of $\mathrm{NCG} / \mathrm{p}-\mathrm{Si}$ Schottky junction based sensor measured at $0 \%, 25 \%, 50 \%, 75 \%$ and $100 \%$ methane concentration at room temperature

Table 1. The reverse Barrier Height, $\emptyset_{b(R E V)}$ of NCG/p-Si Schottky Junction with Various Concentrations of $\mathrm{CH}_{4}$ at Room Temperature

\begin{tabular}{cc}
\hline $\mathrm{CH}_{4}(\%)$ & $\emptyset_{b(\text { REV })}(\mathrm{eV})$ \\
\hline air ambient & 0.58 \\
25 & 0.56 \\
50 & 0.56 \\
75 & 0.54 \\
100 & 0.53 \\
\hline
\end{tabular}


Table 1 indicates the reduction of reverse barrier height when the device exposed to a higher concentration of $\mathrm{CH}_{4}$ gas. This phenomenon indicates the adsorption and desorption of gaseous molecules into the surface of the thin film will influence the electrical properties of the devices. It was found that the oxygen ions already exist at the grain boundaries due to the reaction with Oxygen molecules from the surroundings of the metal surface. During the reaction, $\mathrm{CH}_{4}$ undergoes dissociative adsorption and consequently yielding $\mathrm{CH}_{3}$ or $\mathrm{H}$ on the metal surface. Subsequently, these species will react with the chemisorbed ionic oxygen, which resulting additional negative charge carriers to the grain boundaries and increases the current through the junction [1].

\section{CONCLUSION}

In this paper, the I-V characteristic of the $\mathrm{NCG} / \mathrm{p}-\mathrm{Si}$ were examined under different methane gas concentration at room temperature. The response of the device towards methane gas shown in the reverse bias where the large current shift produced by the device. The value of $\emptyset_{b(R E V)}$ extracted from the plot of $\ln \left(I_{R} / S A^{*} T^{2}\right)=-q \emptyset_{b(R E V)} / k T+(q \alpha / k T)\left|V_{R}\right|^{1 / 4}$, shows the reduction of $\emptyset_{b(R E V)}$ when exposed to a higher concentration of $\mathrm{CH}_{4}$ gas. $\mathrm{CH}_{4}$ gas adsorbed at the metal surface thus generates negative charge carriers that increases the reverse current density.

\section{ACKNOWLEDGEMENTS}

The authors gratefully acknowledged to the Research University grant GUP Tier 1, vot no. 14H63 and GUP Tier 1, vot no. $19 \mathrm{H} 33$ for the financial sponsor, and would like to thank the Southampton Nanofabrication Centre and MIMOS Berhad for the collaborative work. Also thanks to the Universiti Teknologi Malaysia (UTM) for providing an excellent research environment in which to complete this work.

\section{REFERENCES}

[1] S. Basu and P. K. Basu, "Nanocrystalline metal oxides for methane sensors: Role of noble metals," J. Sensors, vol. 2009, pp. 81-88, 2009.

[2] R. Bogue, "Nanomaterials for gas sensing: a review of recent research,” Sensor. Review, vol. 34, pp. 1-8, 2014.

[3] G. Jiménez-Cadena, J. Riu, and F. X. Rius, "Gas sensors based on nanostructured materials.," Analyst, vol. 132, no. 11, pp. 1083-1099, 2007.

[4] H. Elhaes, A. Fakhry, and M. Ibrahim, "Carbon nano materials as gas sensors," Mater. Today Proc., vol. 3, no. 6, pp. 2483-2492, 2016.

[5] W. H. Khoo and S. M. Sultan, "A study on the gas sensing effect on current-voltage characteristics of $\mathrm{ZnO}$ nanostructures," IEEE Int. Conf. Semicond. Electron. Proceedings, ICSE, vol. 1, pp. 221-224, 2014.

[6] D. Kohl, "Function and applications of gas sensors," J. Phys. D. Appl. Phys., vol. 34, no. 19, pp. 125-149, 2001.

[7] P. Shankar, J. Bosco, and B. Rayappan, "Gas sensing mechanism of metal oxides : The role of ambient atmosphere type of semiconductor and gases - A review ScienceJet," Science Letters Journal, vol. 4, pp. 126, 2015.

[8] G. F. Fine, L. M. Cavanagh, A. Afonja, and R. Binions, "Metal oxide semi-conductor gas sensors in environmental monitoring," Sensors, vol. 10, no. 6, pp. 5469-5502, 2010.

[9] S. J. Fishlock, D. Grech, J. W. McBride, H. M. H. Chong, and S. H. Pu, "Mechanical characterisation of nanocrystalline graphite using micromechanical structures," Microelectron. Eng., vol. 159, pp. 184-189, 2016.

[10] G. Chimowa et al., "Improving methane gas sensing properties of multi-walled carbon nanotubes by vanadium oxide filling," Sensors Actuators, vol. 247, pp. 11-18, 2017.

[11] Q. Wang and B. Arash, "A review on applications of carbon nanotubes and graphenes as nano-resonator sensors," Comput. Mater. Sci., vol. 82, pp. 350-360, 2014.

[12] C. I. L. Justino, A. R. Gomes, A. C. Freitas, A. C. Duarte, and T. A. P. Rocha-Santos, "Graphene based sensors and biosensors," TrAC - Trends in Analytical Chemistry, vol. 91, pp. 53-66, 2017.

[13] M. Shafiei et al., "Reversed bias Pt/nanostructured $\mathrm{ZnO}$ Schottky diode with enhanced electric field for hydrogen sensing," Sensors Actuators, B Chem., vol. 146, no. 2, pp. 507-512, 2010.

[14] J. Yu, S. J. Ippolito, M. Shafiei, D. Dhawan, W. Wlodarski, and K. Kalantar-Zadeh, "Reverse biased Pt/nanostructured $\mathrm{MoO}_{3} / \mathrm{SiC}$ Schottky diode based hydrogen gas sensors," Appl. Phys. Lett., vol. 94, no. 1, pp. 2007-2010, 2009.

[15] J. Yu, S. J. Ippolito, W. Wlodarski, M. Strano, and K. Kalantar-Zadeh, "Nanorod based Schottky contact gas sensors in reversed bias condition," Nanotechnology, vol. 21, no. 26, pp. 1-8, 2010.

[16] A. Singh, T. Sudarshan, and G. Koley, "Tunable Reverse-Biased Graphene / Silicon Heterojunction Schottky Diode Sensor," Nano Micro Small Journal., vol. 10, no. 8, pp. 1555-1565, 2014.

[17] L. E. Mitchell, E. J. Brook, T. Sowers, J. R. McConnell, and K. Taylor, "Multidecadal variability of atmospheric methane, 1000-1800 C.E.," J. Geophys. Res. Biogeosciences, vol. 116, no. 2, pp. 1-16, 2011.

[18] E. Llobet, "Gas sensors using carbon nanomaterials: A review," Sensors Actuators, B Chem., vol. 179, pp. $32-45,2013$. 
[19] M. V. S. Chandrashekhar, J. Lu, M. G. Spencer, M. Qazi, and G. Koley, "Large area nanocrystalline graphite films on $\mathrm{SiC}$ for gas sensing applications," Proc. IEEE Sensors, vol. 12, pp. 558-561, 2007.

[20] S. M. Sultan, S. H. Pu, S. J. Fishlock, L. H. Wah, and S. Member, "Electrical behavior of Nanocrystalline graphite / p-Si Schottky Diode," IEEE 16th Int. Conf. Nanotechnol., no. August 22-25, pp. 307-310, 2016.

[21] A. A. Nawawi, S. M. Sultan, S. F. A. Rahman, S. H. Pu, J. W. McBride, and L. H. Wah, "A study of different extraction techniques of nanocrystalline graphite (NCG)/p-type silicon schottky diode parameters," Proc. 2017 IEEE Reg. Symp. Micro Nanoelectron. RSM 2017, no. 14, pp. 119-122, 2017.

[22] H. Medina et al., "Metal-free growth of nanographene on silicon oxides for transparent conducting applications," Adv. Funct. Mater., vol. 22, no. 10, pp. 2123-2128, 2012.

[23] A. C. Ferrari, "Raman spectroscopy of graphene and graphite : Disorder, electron - phonon coupling, doping and nonadiabatic effects," Solid State Communications, vol. 143, pp. 47-57, 2007.

[24] S. M. Sze and Kwok K. Ng, "Physics of Semiconductor Devices 3rd Edition," John Wiley \& Sons, Inc., Hoboken, New Jersey, 2007.

[25] J. S. H. Elhadidy, "Symmetrical current - voltage characteristic of a metal - semiconductor - metal structure of Schottky contacts and parameter retrieval of a CdTe structure," Semiconductor Science and Technology, IOP Publishing, vol. 27, pp. 1-6, 2015. 